\title{
Real-World Utilization and Safety of Daratumumab IV Rapid Infusions Administered in a Community Setting: A Retrospective Observational Study
}

\author{
Lucio Gordan ${ }^{1} \cdot$ Melody Chang ${ }^{1} \cdot$ Marie-Hélène Lafeuille ${ }^{2} \cdot$ Hela Romdhani $^{2} \cdot$ Fuad Paramasivam $^{2} \cdot$ Eric M. Maiese $^{3}$. \\ Caroline McKay ${ }^{3}$
}

Accepted: 29 December 2020 / Published online: 9 February 2021

(c) The Author(s) 2021

\begin{abstract}
Background Some institutions have implemented a daratumumab intravenous rapid-infusion protocol in which patients with multiple myeloma (MM) receive their third and subsequent infusions within $\sim 90$ min instead of $\geq 3 \mathrm{~h}$.

Objective This study sought to understand the utilization, effectiveness, and infusion reactions (IRs) observed in patients with MM who received daratumumab rapid infusions.

Methods Electronic medical records from Florida Cancer Specialists \& Research Institute were used. Adult patients with MM who received one or more rapid daratumumab infusion (full dose in $\leq 110 \mathrm{~min}$ ) at their third or later infusion of the first daratumumab-containing regimen (index date: 16 November 2015 to 15 March 2019) were included. IRs included events that (1) occurred $\leq 24 \mathrm{~h}$ post-daratumumab infusion or (2) were stated as an IR in the patient charts. Non-IR adverse events (AEs) were events attributed to daratumumab in patient charts that did not meet the IR definition.

Results In total, 147 patients received one or more rapid infusion in their first daratumumab-containing regimen. Median time from initial MM diagnosis to index date was 2.5 years. Non-IR AEs occurred in $10.2 \%$ of patients during treatment, and $36.7 \%$ experienced one or more IR after receiving a daratumumab infusion. No IRs occurred after a rapid infusion. The overall response rate was $91.1 \%$ (after rapid infusions only: $71.3 \%$ ).

Conclusions This study provides real-world evidence on the practice patterns of daratumumab rapid infusions in a large community-based oncology clinic system. These results suggest that treatment regimens including daratumumab rapid infusions at the third infusion or later were well-tolerated, and their effectiveness was comparable to that observed in clinical trials.
\end{abstract}

Supplementary Information The online version contains supplementary material available at https://doi.org/10.1007/s4080 1-020-00226-3.

Marie-Hélène Lafeuille

Marie-Helene.Lafeuille@analysisgroup.com

1 Florida Cancer Specialists, Gainesville, FL, USA

2 Analysis Group, Inc., 1190 avenue des Canadiens-de-Montreal, Tour Deloitte Suite 1500, Montreal, QC H3B 0G7, Canada

3 Janssen Scientific Affairs, LLC, Horsham, NJ, USA

\section{Key Points}

This observational study aimed to assess the real-world utilization, effectiveness, and infusion reactions in patients with multiple myeloma who received daratumumab rapid infusions.

The results suggest that the use of daratumumab rapid infusions at the third infusion or later is well-tolerated and that its effectiveness is comparable to that observed in clinical trials for standard daratumumab infusions. 


\section{Introduction}

Multiple myeloma (MM) is a hematological neoplasm of terminally differentiated plasma cells whose key clinical manifestations are driven by tumor cells infiltrating the bone marrow $[1,2]$. MM is expected to account for $1.8 \%$ of all new cancer cases and for $2.1 \%$ of all cancer deaths in the USA in 2019 [3]. The median overall survival from initiation of MM treatment is approximately 6.9 years in patients with stage II MM and 3.6 years in patients with stage III based on the Revised International Staging System [4].

Current treatment guidelines for MM recommend a sequence of induction therapy followed by autologous stem cell transplantation (in transplant-eligible patients only) and maintenance therapy $[5,6]$. Treatment options for MM typically consist of combinations of proteasome inhibitors (PIs) and immunomodulatory drugs (IMiDs), as well as chemotherapeutic agents [1]. Daratumumab, a monoclonal antibody directed against cluster of differentiation (CD)-38, is one of the newest treatments for MM. It was first approved in 2015 by the US FDA as monotherapy in patients with $\mathrm{MM}$ who had received three or more prior lines of therapy [7-10], and subsequently as combination therapy with a PI and/or IMiD in newly diagnosed patients and in patients with relapsed/refractory (RR) disease [7, 11-14].

Across several trials, infusion reactions (IRs) associated with daratumumab were reported in approximately half of all patients and generally consisted of respiratory symptoms (e.g., bronchospasm, cough, and dyspnea) [8-14]. IRs were predominantly of grade 1 or 2 and rarely led to treatment discontinuation [8, 9, 11-14]. In addition, the majority of IRs arose during the first infusion $[8,9$, 11-14]: $40 \%$ of patients experienced an IR during the first infusion, $2 \%$ during the second infusion, and $4 \%$ in subsequent infusions [15].

In clinical trials, the first infusions of daratumumab $(16 \mathrm{mg} / \mathrm{kg}$ of body weight) had a median duration of 7 $\mathrm{h}$, whereas the second infusions had a median duration of $4 \mathrm{~h}$ [7]. Third and subsequent infusions were typically administered within $3.5 \mathrm{~h}$ [7]. The low incidence of IRs, particularly after the first few administrations, motivated the development of a rapid-infusion protocol in which a standard dose of daratumumab is administered within 90 min (instead of the $3 \mathrm{~h}$ of a standard infusion) for the third and subsequent infusions [16, 17]. In a prospective study by Barr et al. [16], rapid infusions were well-tolerated by patients who had previously received two or more doses: of the 28 patients enrolled, only one experienced a grade 2 event. Similarly, a recent retrospective chart review study of 73 patients with RR MM treated at the Levine Cancer
Institute (LCI) found that patients who received rapid versus standard infusions of daratumumab had similar rates of IRs, suggesting that rapid infusions of daratumumab were safe and tolerable in patients who had previously received two doses [17]. While these studies focused on the safety of rapid infusions of daratumumab, they did not report on effectiveness outcomes among patients receiving rapid infusions.

To further increase our understanding of the utilization, effectiveness, and safety of rapid infusions of daratumumab, this study sought to describe the real-world daratumumab administration characteristics and treatment outcomes (including rates of non-IR adverse event [AEs] and IRs, and best response) in patients with MM who received rapid infusions of daratumumab. Since such outcomes could be affected by the prior use of daratumumab, this study focused on patients with MM who received rapid infusions in their first daratumumab-containing treatment regimen.

\section{Materials and Methods}

\subsection{Data Source}

Electronic medical records (EMRs) from the Florida Cancer Specialists \& Research Institute (FCS) were retrieved (1 January 2013 to 15 March 2019). FCS is a large independent medical oncology/hematology community practice in the USA. The network consists of nearly 100 locations in Florida, involving over 230 physicians [18]. The EMR system included information on patient characteristics, prescription medications, and medical procedures. Information on nonIR AEs, IRs, inpatient stays, emergency room (ER) visits, and treatment response were also collected via manual data extraction from the patient charts. Patient-level information was aggregated via an encrypted patient-unique identifier to comply with the Health Insurance Portability and Accountability Act.

\subsection{Study Design}

A retrospective observational cohort study design was employed. The index date was defined as the date of administration of the first daratumumab intravenous infusion. The study period spanned between the date of the initial FDA approval of daratumumab for MM (16 November 2015) and end of data availability (15 March 2019). Treatment regimens were constructed based on all antineoplastic agents initiated post-MM diagnosis. A 30-day window after the first record of an antineoplastic agent post-MM diagnosis was used to determine all agents included in the first MM treatment regimen received. A regimen ended at the earliest of (1) initiation of a new antineoplastic agent that is not part of 
the initial regimen or (2) discontinuation of all the agents of the regimen (i.e., a gap of at least 90 days between prescriptions/infusions or between the last prescription and the end of data availability). Treatment regimens with no evidence of termination were censored at the end of data availability. The index treatment regimen was defined as the patient's first daratumumab-containing regimen.

\subsection{Selection Criteria}

Patients were included in the analysis if they met the following criteria: (1) one or more administration of daratumumab; (2) one or more diagnosis of MM (International Classification of Diseases, Ninth Revision, Clinical Modification [ICD-9-CM]: 203.xx; ICD-10-CM: C90.xx) prior to or on the index date; (3) aged $\geq 18$ years as of the index date; (4) clinically active in the FCS network (two or more visits at an FCS facility); (5) no participation in a daratumumab clinical trial (i.e., no daratumumab administered before 16 November 2015 and no indication of use of "research daratumumab" or of a clinical trial setting in the data); (6) one or more full intravenous dose of daratumumab (defined as $\geq 90 \%$ of the dose ordered by the physician administered for which the dosage corresponded to the FDA-approved dose [i.e., $\geq 80 \%$ of the calculated dose assuming a body weightadjusted dosage of $16 \mathrm{mg} / \mathrm{kg}$ ]) [7]; (7) one or more rapid infusion defined as a full infusion (as defined in selection criterion 6) for which the length of daratumumab administration lasted $\leq 110 \mathrm{~min}$ (instead of $90 \mathrm{~min}$, to allow for variability in administration duration observed in the EMR); and (8) one or more rapid infusion in the first daratumumabcontaining treatment regimen (i.e., the index regimen), with the first rapid infusion being the third or later daratumumab intravenous infusion observed.

\subsection{Study Measures and Statistical Analysis}

Patient demographic characteristics were assessed on the index date and included age, sex, ethnicity, and year of index date. Clinical characteristics, including time from the initial MM diagnosis to the index date, MM stage (according to the International Staging System) prior to or on the index date, Eastern Cooperative Oncology Group (ECOG) score as of the index date, and the number of prior antineoplastic treatment regimens received, were reported.

In addition to the agents included in the index treatment regimen and days on daratumumab treatment, administration characteristics were assessed. These included the total number of infusions and the number of rapid infusions administered per patient, the infusion number of the first observed rapid infusion, and the duration of rapid infusions.

Treatment outcomes included the occurrence of non-IR AEs and IRs during the index treatment regimen and best response to daratumumab. IRs included reactions, signs, and symptoms that either (1) occurred within $24 \mathrm{~h}$ of a daratumumab intravenous infusion or (2) were explicitly stated as an IR based on the patient's charts. IRs attributed to any daratumumab intravenous infusions (standard or rapid) during the index treatment regimen were reported, as well as the subset of IRs attributed to rapid infusions. Non-IR AEs were defined as health events explicitly attributed to daratumumab in the patient charts that did not also meet the definition of an IR. Hospitalizations and ER visits that occurred within 24 $\mathrm{h}$ of a daratumumab intravenous infusion were also reported among all daratumumab intravenous infusions during the index treatment regimen, and among the subset of rapid infusions. Best response to daratumumab was reported as per physician's assessment during regular clinical care.

Means, standard deviations (SDs), medians, and interquartile ranges (IQRs) were used to report continuous variables. Counts and percentages were used to report categorical variables.

\section{Results}

\subsection{Patient Demographic and Clinical Characteristics}

Of 534 patients with MM who received one or more full intravenous infusion of daratumumab, 147 (27.5\%) received one or more rapid infusion during their first daratumumabcontaining regimen and were included in the analysis (Fig. 1). Patients' median age was 73.0 years (mean 71.9 \pm 9.0; IQR 13.0) as of the index date, and 66 (44.9\%) were females (Table 1). The index date was in 2016 for 15 (10.2\%) patients, in 2017 for 70 (47.6\%) patients, in 2018 for 61 (41.5\%) patients, and in 2019 for one (0.7\%) patient. Patients initiated daratumumab within a median of 2.5 years (mean $3.4 \pm 4.3$; IQR 3.4) after the MM diagnosis. ECOG performance status was $\leq 2$ for $136(92.5 \%)$ patients, including 39 (26.5\%) with an ECOG score of 0, 82 (55.8\%) with an ECOG score of 1, and $15(10.2 \%)$ with a score of 2 . Patients received a median of 2 (mean $2.5 \pm 2.1$; IQR 3 ) antineoplastic treatment regimens prior to the index date. A total of 19 (12.9\%) patients had not received any antineoplastic treatment regimen prior to the index date (Table 1).

\subsection{Administration Characteristics}

The most common daratumumab-containing regimens were daratumumab monotherapy $(\mathrm{N}=50[34.0 \%])$ and daratumumab in combination with pomalidomide $(\mathrm{N}=49$ [33.3\%]). Other regimens included daratumumab in combination with lenalidomide ( $\mathrm{N}=20[13.6 \%])$, bortezomib $(\mathrm{N}=13$ [8.8\%]), or other agents $(\mathrm{N}=15[10.2 \%])$. During the index treatment 


\begin{tabular}{|c|c|}
\hline \multicolumn{2}{|c|}{$\begin{array}{l}\qquad \begin{array}{l}\geq 1 \text { administration of daratumumab } \\
\text { The date of the first daratumumab administration is the index date } \\
\qquad \mathbf{N}=\mathbf{5 7 5}\end{array}\end{array}$} \\
\hline \multicolumn{2}{|c|}{ Initial MM diagnosis prior to or on the index date ${ }^{1}$} \\
\hline $\mathbf{N}=\mathbf{5 7 4}$ & (99.8\%) \\
\hline \multicolumn{2}{|c|}{$\geq 18$ years of age } \\
\hline $\mathbf{N}=574$ & $(100.0 \%)$ \\
\hline \multicolumn{2}{|c|}{ Clinically active in FCS network, defined as having $\geq 2$ visits at an FCS facility } \\
\hline $\mathbf{N}=\mathbf{5 7 3}$ & $(99.8 \%)$ \\
\hline \multicolumn{2}{|c|}{ No participation in a daratumumab clinical trial at any time } \\
\hline $\mathbf{N}=551$ & $(96.2 \%)$ \\
\hline \multicolumn{2}{|c|}{$\geq 1$ full IV infusion of daratumumab ${ }^{2}$} \\
\hline $\mathbf{N}=534$ & $(99.6 \%)$ \\
\hline \multicolumn{2}{|c|}{$\geq 1$ rapid infusion } \\
\hline \multicolumn{2}{|c|}{$\geq 1$ infusion that is full dose ${ }^{2}$ and administered in $\leq 110$ minutes } \\
\hline $\mathbf{N}=\mathbf{2 2 7}$ & (42.5\%) \\
\hline \multicolumn{2}{|c|}{$\begin{array}{l}\geq 1 \text { rapid infusion in the first daratumumab-containing treatment regimen received } \\
\text { (first rapid infusion being the } 3^{\text {rd }} \text { or later daratumumab IV infusion observed) }\end{array}$} \\
\hline$N=147$ & $(64.8 \%)$ \\
\hline
\end{tabular}

Fig. 1 Sample selection. Data source: Electronic medical records from Florida Cancer Specialists \& Research Institute (1 Jan 2013-15 Mar 2019). ${ }^{1}$ The date of the diagnosis could be prior to 1 January 2013. ${ }^{2}$ Defined as at least one complete IV infusion of daratumumab, (i.e., an infusion for which $90 \%$ of the dose ordered by the physician

regimen (median duration 323 days; mean $373.3 \pm 251.1$; IQR 368), a median of 21 daratumumab intravenous infusions (mean $22.1 \pm 10.8$; IQR 14) were administered per patient, including a median of seven (mean $8.1 \pm 5.4$; IQR 8 ) rapid infusions (Table 2). The median first daratumumab intravenous rapid infusion was the 11th infusion (mean $12.8 \pm 9.3$; IQR 15) (Table 2). Among all rapid infusions $(\mathrm{N}=1185)$, the median duration of daratumumab intravenous infusion was 92 min (mean $92.9 \pm 8.6$; IQR 9), with 497 (41.9\%) infusions administered within $\leq 90 \mathrm{~min}, 489(41.3 \%)$ between 91 and $100 \mathrm{~min}$, and 199 (16.8\%) between 101 and $110 \mathrm{~min}$ (Table 2). was administered to the patient) for which the dosage corresponded to the FDA-approved dose (i.e., administration of $\geq 80 \%$ of the calculated dose assuming a body weight-adjusted dosage of $16 \mathrm{mg} / \mathrm{kg}$ ). FCS Florida Cancer Specialist, IV intravenous, $M M$ multiple myeloma

\subsection{Daratumumab Treatment Outcomes}

\subsubsection{Non-Infusion Reaction Adverse Events}

Among the 15 (10.2\%) patients who experienced one or more non-IR AE during the index treatment regimen, the median number of non-IR AEs per patient was two (mean $1.9 \pm 1.2$; IQR 2). General disorders and administration site conditions were observed in eight $(5.4 \%)$ patients, all of whom had fatigue and one of whom $(0.7 \%)$ had pyrexia. Four patients $(2.7 \%)$ had gastrointestinal toxicities, including two (1.4\%) with vomiting, two (1.4\%) with nausea, one $(0.7 \%)$ with constipation, and one $(0.7 \%)$ with diarrhea (Table 1 in the electronic supplementary material). 
Table 1 Demographic and clinical characteristics in patients with multiple myeloma with at least one rapid infusion during their first daratumumabcontaining treatment regimen

\begin{tabular}{|c|c|}
\hline Characteristics & $\mathrm{N}=147$ \\
\hline \multicolumn{2}{|l|}{ Demographic characteristics $^{a}$} \\
\hline Age (years), mean \pm SD [median, IQR] & $71.9 \pm 9.0[73.0 ; 13.0]$ \\
\hline Female sex, $n(\%)$ & $66(44.9)$ \\
\hline \multicolumn{2}{|l|}{ Ethnicity, $n(\%)$} \\
\hline Hispanic or Latino & $8(5.4)$ \\
\hline Not Hispanic or Latino & $131(89.1)$ \\
\hline Unknown & $8(5.4)$ \\
\hline \multicolumn{2}{|l|}{ Year of index date $^{\mathrm{b}}, n(\%)$} \\
\hline 2015 & $0(0.0)$ \\
\hline 2016 & $15(10.2)$ \\
\hline 2017 & $70(47.6)$ \\
\hline 2018 & $61(41.5)$ \\
\hline 2019 & $1(0.7)$ \\
\hline \multicolumn{2}{|l|}{ Clinical characteristics } \\
\hline $\begin{array}{l}\text { Time between initial MM diagnosis and index date (years), mean } \pm \text { SD [median, } \\
\text { IQR] }\end{array}$ & $3.4 \pm 4.3[2.5 ; 3.4]$ \\
\hline \multicolumn{2}{|l|}{ MM stage ${ }^{\mathrm{c}}, n(\%)$} \\
\hline ISS Stage I & $11(7.5)$ \\
\hline ISS Stage II & $20(13.6)$ \\
\hline ISS Stage III & $46(31.3)$ \\
\hline Unknown & $70(47.6)$ \\
\hline \multicolumn{2}{|l|}{ ECOG performance status ${ }^{\mathrm{a}}, n(\%)$} \\
\hline 0 & $39(26.5)$ \\
\hline 1 & $82(55.8)$ \\
\hline 2 & $15(10.2)$ \\
\hline 3 & $3(2.0)$ \\
\hline Unknown & $8(5.4)$ \\
\hline Number of prior antineoplastic treatment regimens ${ }^{\mathrm{d}}$, mean \pm SD [median, IQR] & $2.5 \pm 2.1[2 ; 3]$ \\
\hline One or more prior treatment regimen & $128(87.1)$ \\
\hline No prior treatment regimen & $19(12.9)$ \\
\hline
\end{tabular}

Data are presented as mean \pm standard deviation [median; interquartile range] or $\mathrm{N}(\%)$ unless otherwise indicated

ECOG Eastern Cooperative Oncology Group, ISS International Staging System, MM multiple myeloma

${ }^{a}$ Measured on the index date

${ }^{\mathrm{b}}$ The study period ranged from 16 November 2015 to 15 March 2019

${ }^{\mathrm{c}}$ Measured prior to or on the index date

${ }^{\mathrm{d}}$ Anytime prior to the index date

\subsubsection{Infusion Reactions}

Of the 147 patients included in this study, 54 (36.7\%) experienced one or more IR after receiving a daratumumab intravenous infusion during the index treatment regimen: 52 (35.4\%) experienced an IR after receiving their first daratumumab dose, one $(0.7 \%)$ after receiving their second dose, and six (4.1\%) after receiving their third or later dose (patients could experience IRs after more than one dose). The median number of IRs per patient (i.e., individual symptoms) among patients with one or more IR was two (mean $2.5 \pm 1.3$; IQR 1) (Table 2). The most commonly reported IRs were nausea in $17(11.6 \%)$ patients, chills in $14(9.5 \%)$ patients, and dyspnea in $11(7.5 \%)$ patients (Table 2). Of these 54 patients, only one $(0.7 \%)$ had a hospitalization within $24 \mathrm{~h}$ of an IR, in which the planned dose was $1400 \mathrm{mg}$ but only $280 \mathrm{mg}$ was administered (interrupted dose). No patient had an ER visit associated with a daratumumab intravenous infusion (Table 2). No patient experienced an IR after receiving a rapid daratumumab intravenous infusion (Table 2). 
Table 2 Daratumumab administration characteristics and infusion reactions

\begin{tabular}{|c|c|c|}
\hline Characteristics and infusion reactions & $\mathrm{N}=147$ & \\
\hline \multicolumn{3}{|l|}{ DAR administration characteristics } \\
\hline DAR IV infusions per patient, mean \pm SD [median, IQR] & \multicolumn{2}{|l|}{$22.1 \pm 10.8[21 ; 14]$} \\
\hline DAR IV rapid infusions per patient, mean $\pm \mathrm{SD}$ [median, IQR] & \multicolumn{2}{|l|}{$8.1 \pm 5.4[7 ; 8]$} \\
\hline Infusion number of the first observed rapid infusion, mean $\pm \mathrm{SD}$ [median, IQR] & \multicolumn{2}{|l|}{$12.8 \pm 9.3[11 ; 15]$} \\
\hline \multicolumn{3}{|l|}{ Infusion number of the first observed rapid infusion, $n(\%)$} \\
\hline 3rd administration & \multicolumn{2}{|l|}{$24(16.3)$} \\
\hline 4th-10th administration & \multicolumn{2}{|l|}{$49(33.3)$} \\
\hline 11th-20th administration & \multicolumn{2}{|l|}{$42(28.6)$} \\
\hline 20th-30th administration & \multicolumn{2}{|l|}{$24(16.3)$} \\
\hline 31 st administration or later & \multicolumn{2}{|l|}{$8(5.4)$} \\
\hline Duration of DAR IV rapid infusions (minutes), mean $\pm \mathrm{SD}$ [median, IQR] & \multicolumn{2}{|l|}{$92.9 \pm 8.6[92 ; 9]$} \\
\hline \multicolumn{3}{|l|}{ Number of DAR IV rapid infusions by infusion duration (minutes), $n(\%)$} \\
\hline$\leq 70$ & \multicolumn{2}{|l|}{$17(1.4)$} \\
\hline $71-80$ & \multicolumn{2}{|l|}{$39(3.3)$} \\
\hline $81-90$ & \multicolumn{2}{|l|}{$441(37.2)$} \\
\hline $91-100$ & \multicolumn{2}{|l|}{$489(41.3)$} \\
\hline $101-110$ & \multicolumn{2}{|l|}{$199(16.8)$} \\
\hline Total number of rapid infusions & \multicolumn{2}{|l|}{$\mathrm{N}=1185$} \\
\hline Days on DAR within the treatment regimen, mean $\pm \mathrm{SD}$ [median, IQR] & \multicolumn{2}{|l|}{$373.3 \pm 251.1[323 ; 368]$} \\
\hline DAR IV infusion reactions ${ }^{a}$ & Among all infusions & Among rapid infusions only \\
\hline Number of patients with one or more IR, $n(\%)$ & $54(36.7)$ & $0(0.0)$ \\
\hline At the 1st administration & $52(35.4)$ & - \\
\hline At the 2nd administration & $1(0.7)$ & - \\
\hline At the 3rd administration or later & $6(4.1)$ & - \\
\hline \multicolumn{3}{|l|}{ Among patients whose first observed rapid infusion occurred on the } \\
\hline 3rd administration & $5(3.4)$ & - \\
\hline 4th-10th administration & $19(12.9)$ & - \\
\hline$\geq 11$ th administration & $30(20.4)$ & - \\
\hline Number of IRs per patient ${ }^{\mathrm{b}}$, mean $\pm \mathrm{SD}$ [median, IQR] & $2.5 \pm 1.3[2 ; 1]$ & - \\
\hline \multicolumn{3}{|l|}{ IRs, $n(\%)$} \\
\hline \multicolumn{3}{|l|}{ Severe reactions } \\
\hline Bronchospasm & $1(0.7)$ & $0(0.0)$ \\
\hline Hypoxia & $0(0.0)$ & $0(0.0)$ \\
\hline Dyspnea & $11(7.5)$ & $0(0.0)$ \\
\hline Hypertension & $3(2.0)$ & $0(0.0)$ \\
\hline Laryngeal edema & $0(0.0)$ & $0(0.0)$ \\
\hline Pulmonary edema & $0(0.0)$ & $0(0.0)$ \\
\hline Common signs and symptoms & & \\
\hline Chills & $14(9.5)$ & $0(0.0)$ \\
\hline Nausea & $17(11.6)$ & $0(0.0)$ \\
\hline Respiratory symptoms ${ }^{\mathrm{c}}$ & $2(1.4)$ & $0(0.0)$ \\
\hline Vomiting & $9(6.1)$ & $0(0.0)$ \\
\hline Other symptoms & & \\
\hline Abdominal discomfort & $1(0.7)$ & $0(0.0)$ \\
\hline Chest discomfort & $7(4.8)$ & $0(0.0)$ \\
\hline Cough & $8(5.4)$ & $0(0.0)$ \\
\hline Diarrhea & $0(0.0)$ & $0(0.0)$ \\
\hline Ear discomfort & $0(0.0)$ & $0(0.0)$ \\
\hline Epiphora & $3(2.0)$ & $0(0.0)$ \\
\hline Fatigue & $2(1.4)$ & $0(0.0)$ \\
\hline
\end{tabular}


Table 2 (continued)

\begin{tabular}{|c|c|c|}
\hline Characteristics and infusion reactions & $\mathrm{N}=147$ & \\
\hline Flush & $5(3.4)$ & $0(0.0)$ \\
\hline Headache & $3(2.0)$ & $0(0.0)$ \\
\hline Heart palpitations & $0(0.0)$ & $0(0.0)$ \\
\hline Hives & $0(0.0)$ & $0(0.0)$ \\
\hline Infiltration & $2(1.4)$ & $0(0.0)$ \\
\hline Itching & $3(2.0)$ & $0(0.0)$ \\
\hline Lightheadedness & $1(0.7)$ & $0(0.0)$ \\
\hline Back pain & $4(2.7)$ & $0(0.0)$ \\
\hline Muscle spasm & $1(0.7)$ & $0(0.0)$ \\
\hline Oral discomfort & $1(0.7)$ & $0(0.0)$ \\
\hline Rhinorrhea & $9(6.1)$ & $0(0.0)$ \\
\hline Rigors & $3(2.0)$ & $0(0.0)$ \\
\hline Sneeze & $0(0.0)$ & $0(0.0)$ \\
\hline Sweating & $1(0.7)$ & $0(0.0)$ \\
\hline Throat discomfort & $4(2.7)$ & $0(0.0)$ \\
\hline Tremors & $2(1.4)$ & $0(0.0)$ \\
\hline Wheezing & $3(2.0)$ & $0(0.0)$ \\
\hline Other $^{\mathrm{d}}$ & $11(7.5)$ & $0(0.0)$ \\
\hline Patients with at least one visit within $24 \mathrm{~h}$ of a DAR IV infusion reaction, $n(\%)$ & $1(0.7)$ & - \\
\hline Hospitalization & $1(0.7)$ & - \\
\hline ER visit & $0(0.0)$ & - \\
\hline
\end{tabular}

Data are presented as mean \pm standard deviation [median; interquartile range] or $\mathrm{N}(\%)$ unless otherwise indicated

$D A R$ daratumumab, ER emergency room, $I Q R$ interquartile range, $I R$ infusion reaction, $S D$ standard deviation

${ }^{a}$ IRs included reactions, signs, and symptoms that either (1) occurred within $24 \mathrm{~h}$ of an IV infusion of daratumumab or (2) were explicitly stated as an IR based on the patient's charts

${ }^{\mathrm{b}}$ Among patients with at least one IR

${ }^{\mathrm{c}}$ Respiratory symptoms included shortness of breath and difficulty in breathing

${ }^{\mathrm{d}}$ Other IRs reported included clamminess, dizziness, epigastric pain, extravasation, feeling cold, hypotension, indigestion, joint pain, nonspecific infusion reaction, oxygen desaturation, facial pain, heartburn, and tingling in the upper extremities

\subsubsection{Best Response}

Information on best response recorded during the index treatment regimen was available for $123(83.7 \%)$ patients. Among these, no patient had a stringent complete response, seven $(4.8 \%)$ had a complete response, nine $(6.1 \%)$ had a very good partial response, $15(10.2 \%)$ had a partial response, and $81(55.1 \%)$ had a response of unknown depth, yielding an overall response rate (ORR) of $91.1 \%$. Best recorded response was "stable disease" in seven (4.8\%) patients and "disease progressed" in four $(2.7 \%)$ patients. When looking specifically for responses recorded after the first rapid infusion, the ORR observed was $71.3 \%$.

\section{Discussion}

In this observational real-world study, of 534 patients who received at least one full intravenous infusion of daratumumab in the FCS network, $147(27.5 \%)$ received at least one rapid intravenous infusion of daratumumab during their first daratumumab-containing treatment regimen, with the first rapid infusion being the third or later daratumumab intravenous infusion observed. The median duration of a rapid infusion was $92 \mathrm{~min}$. The ORR was $91.1 \%$, and approximately $10 \%$ of patients experienced at least one non-IR AE during the treatment regimen. IRs after receipt of daratumumab intravenous infusions occurred in $36.7 \%$ of patients, and no patients experienced IRs after receiving a rapid infusion.

The present study used a threshold of 110 min to identify rapid infusions of a full dose of daratumumab. This threshold was chosen based on the observed distribution of infusion durations in the sample and served to account for variation in infusion durations in the real world. Although this threshold is slightly higher than the 90-min definition of rapid infusions used in other studies [16, 17], such a duration remains much shorter than that of a standard infusion (i.e., 3-7 h). 
In this study, no patients experienced an IR after receiving a rapid infusion. This, along with the low rate of nonIR AEs, suggests that rapid infusions are well-tolerated. This is consistent with the findings of the prospective single-arm study by Barr et al. [16], in which rapid infusions of daratumumab were also well-tolerated by all 28 patients enrolled. In the retrospective chart review study by Hamadeh et al. [17], which assessed the safety of rapid infusions of daratumumab in 73 patients with RR MM at LCI, no statistically significant difference was observed between the IR rates in patients who received rapid infusions versus those receiving regular infusions of daratumumab. While the rate of IRs observed for all infusions was $36.7 \%$, no IR was observed for rapid infusions, in the current study. This might be explained by the fact that, by recommendation, rapid infusions were administered at the third or later infusion and that IRs are uncommon after the second infusion of daratumumab [8, 9, 11-14].

In the present study, nearly one-third of patients treated with a daratumumab-containing regimen received at least one rapid infusion. This mode of administration may help reduce the time that patients spend in the treatment setting and would optimize the utilization of healthcare resources [16] without compromising effectiveness. In addition, this mode of administration may be more convenient for patients given the shorter infusion durations. In other disease areas, patients who received rapid infusions expressed a high degree of satisfaction with this improved mode of administration [19, 20].

ORRs observed in previous trials of daratumumab varied depending on the number of prior lines of therapy and the type of daratumumab-containing regimen received. ORRs were lowest in trials of daratumumab monotherapy among patients with RR MM for whom multiple lines of therapy failed (ORRs 29.2 and 35.7\%) [8, 9], higher in trials of daratumumab-containing combination therapies among patients with RR disease for whom at least one line of therapy failed (ORRs 82.9 and 92.9\%) [11, 14], and highest in trials of daratumumab-containing combination therapies in newly diagnosed patients (ORRs 90.9 and $92.6 \%)[12,13]$. In the current study, patients had a median of two prior treatment regimens, and around two-thirds of them received daratumumab as part of a combination therapy. The ORR of $91.1 \%$ observed is thus consistent with findings from trials of daratumumab-containing combination therapies. As expected, the ORR was lower when evaluating responses recorded after the first rapid infusion $(71.3 \%)$, which may be attributed to the later disease stage at which such responses were observed. Moreover, these responses were recorded in the real world, where the selection of patients and clinical follow-up are less regulated than the highly protocolized conditions of a clinical trial, which could also be reflected in a lower ORR.
Taken together, these results suggest that rapid daratumumab infusions are safe and effective. In addition, it could be hypothesized that a wider implementation of the rapid-infusion protocol could lead to important efficiency gains in the delivery of healthcare services. Rapid infusion of daratumumab, along with the recently approved subcutaneous formulation [21], may provide patients with a better experience of treatment.

\subsection{Limitations}

The present study is subject to some limitations. First, the data source drew only from patients clinically active in the FCS network, which may reduce the generalizability of the findings. Second, the study was subject to the limitations of observational studies, including potential data omissions, inconsistent coding practices, and coding errors. For instance, it is possible that data entry errors for patients weight, daratumumab dose, or infusion duration resulted in an inaccurate assessment of daratumumab doses and potentially confounding split or interrupted doses with rapid infusions. Third, information on pre- and postinfusion medication use (e.g., corticosteroids, paracetamol, and antihistamines) was inconsistently reported in the EMR system and thus not reported in this study. Fourth, information on receipt of stem cell transplant was not available in the data. Therefore, the number of prior lines of therapy that the patients received could not be verified. Fifth, daratumumab treatment received outside of the FCS network was not captured in the data, so the rank of daratumumab administrations observed in the data may not reflect accurately the actual number of daratumumab administrations received by the patient. Lastly, non-IR AEs and IRs were extracted from patient charts and may be underreported. In addition, the severity of non-IR AEs and IRs assessed with the National Cancer Institute Common Terminology Criteria for AEs [22] was mostly unknown, so only the incidence of non-IR AEs and IRs was reported, and not their severity.

\section{Conclusion}

This retrospective study provides evidence on real-world practice patterns of daratumumab in a large communitybased clinic system. Initiation of daratumumab intravenous rapid infusions at the third infusion or later among patients with MM was well-tolerated. The effectiveness of treatment regimens containing such rapid infusions was comparable to that observed in clinical trials.

Acknowledgements Medical writing assistance was provided by Samuel Rochette, who is an employee of Analysis Group, Inc., which 
provided paid consulting services to Janssen Scientific Affairs, LLC. for the conduct of the present study.

\section{Declarations}

Conflict of interest LG is president and managing physician at Florida Cancer Specialists \& Research Institute, president of American Oncology Partners, and a board member of Community Oncology Alliance and Florida Society of Clinical Oncology (FLASCO). MC is director of pharmacy operations at Florida Cancer Specialists \& Research Institute, director of pharmacy operations at American Oncology Network, pharmacist liaison at the board of directors of FLASCO, and member of the Oncology Pharmacy Education Network Advisory Committee of the Association of Community Cancer Centers. CM is a former employee of Janssen Scientific Affairs, LLC and may own stocks or stock options. EMM is a former employee of Janssen Scientific Affairs, LLC and may own stocks or stock options. MHL, HR, and FP are employees of Analysis Group, Inc., which provided paid consulting services to Janssen Scientific Affairs, LLC for the conduct of the present study.

Funding This work was supported by Janssen Scientific Affairs, LLC.

Ethics approval Not applicable to this study.

Consent to participate Not applicable to this study.

Consent for publication Not applicable.

Availability of data and material The data used in this study are not publicly available.

\section{Code availability Not applicable.}

Previous presentation Partial results of this study were presented as a published research abstract at the 61st American Society of Hematology Annual Meeting \& Exposition (2019).

Open Access This article is licensed under a Creative Commons Attribution-NonCommercial 4.0 International License, which permits any non-commercial use, sharing, adaptation, distribution and reproduction in any medium or format, as long as you give appropriate credit to the original author(s) and the source, provide a link to the Creative Commons licence, and indicate if changes were made. The images or other third party material in this article are included in the article's Creative Commons licence, unless indicated otherwise in a credit line to the material. If material is not included in the article's Creative Commons licence and your intended use is not permitted by statutory regulation or exceeds the permitted use, you will need to obtain permission directly from the copyright holder. To view a copy of this licence, visit http://creativecommons.org/licenses/by-nc/4.0/.

\section{References}

1. Kumar SK, Rajkumar V, Kyle RA, et al. Multiple myeloma. Nat Rev Dis Primers. 2017;3:17046.

2. Smith D, Yong K. Multiple myeloma. BMJ. 2013;346:f3863.

3. Surveillance Epidemiology and End Results (SEER). Cancer Stat Facts: Myeloma. https://seer.cancer.gov/statfacts/html/mulmy .html.

4. American Cancer Society. Survival Rates by Stage for Multiple Myeloma. 2019. https://www.cancer.org/cancer/multiple-myelo ma/detection-diagnosis-staging/survival-rates.html\#references. Accessed 24 Oct 2019.

5. Moreau P, San Miguel J, Sonneveld P, et al. Multiple myeloma: ESMO Clinical Practice Guidelines for diagnosis, treatment and follow-up. Ann Oncol. 2017;28(Suppl 4):iv52-61.

6. National Comprehensive Cancer Network. NCCN Clinical Practice Guidelines in Oncology-Multiple Myeloma Version 1.2020. 2019. https://www.nccn.org/professionals/physician_gls/pdf/ myeloma.pdf. Accessed 19 Sep 2019.

7. US Food and Drug Administration. Daratumumab (DARZALEX). 2019. https://www.accessdata.fda.gov/drugsatfda_docs/label /2019/761036s024lbl.pdf. Accessed 21 Oct 2019.

8. Lokhorst HM, Plesner T, Laubach JP, et al. Targeting CD38 with daratumumab monotherapy in multiple myeloma. N Engl J Med. 2015;373(13):1207-19.

9. Lonial S, Weiss BM, Usmani SZ, et al. Daratumumab monotherapy in patients with treatment-refractory multiple myeloma (SIRIUS): an open-label, randomised, phase 2 trial. Lancet. 2016;387(10027):1551-60.

10. Chari A, Suvannasankha A, Fay JW, et al. Daratumumab plus pomalidomide and dexamethasone in relapsed and/or refractory multiple myeloma. Blood. 2017;130(8):974-81.

11. Dimopoulos MA, Oriol A, Nahi H, et al. Daratumumab, lenalidomide, and dexamethasone for multiple myeloma. N Engl J Med. 2016;375(14):1319-31.

12. Mateos MV, Dimopoulos MA, Cavo M, et al. Daratumumab plus bortezomib, melphalan, and prednisone for untreated myeloma. N Engl J Med. 2018;378(6):518-28.

13. Moreau P, Attal M, Hulin C, et al. Bortezomib, thalidomide, and dexamethasone with or without daratumumab before and after autologous stem-cell transplantation for newly diagnosed multiple myeloma (CASSIOPEIA): a randomised, open-label, phase 3 study. Lancet. 2019;394(10192):29-38.

14. Palumbo A, Chanan-Khan A, Weisel K, et al. Daratumumab, bortezomib, and dexamethasone for multiple myeloma. N Engl J Med. 2016;375(8):754-66.

15. Janssen CarePath. Dosing \& administration for Darzalex (daratumumab). 2019. https://www.darzalexhcp.com/sites/www.darza lexhcp.com/files/darzalex_dosing_guide1.pdf?v=91. Accessed 1 Sep 2019.

16. Barr H, Dempsey J, Waller A, et al. Ninety-minute daratumumab infusion is safe in multiple myeloma. Leukemia. 2018;32(11):2495-518.

17. Hamadeh IS, Arnall J, Kachur E, et al. Rapid infusion daratumumab is safe and well treated in clinical practice. Blood. 2018;132(Suppl 1):2255.

18. Florida Cancer Specialists \& Research Institute. Our History. https ://flcancer.com/en/your-team/our-history/. Accessed 1 Sep 2019.

19. Daniel SR, McDermott JD Jr, Le C, Pierce CA, Ziskind MA, Ellis LA. A real-world, multi-site, observational study of infusion time and treatment satisfaction with rheumatoid arthritis patients treated with intravenous golimumab or infliximab. J Med Econ. 2018;21(7):724-31.

20. Qazi T, Shah B, El-Dib M, Farraye FA. The tolerability and efficacy of rapid infliximab infusions in patients with inflammatory bowel disease. Dig Dis Sci. 2016;61(2):589-96.

21. US Food \& Drug Administration. Daratumumab (DARZALEX FASPRO). https://www.accessdata.fda.gov/drugsatfda_docs/label /2020/761145s000lbl.pdf. Accessed 4 Dec 2020.

22. National Cancer Institute - Division of Cancer Treatment \& Diagnosis. https://ctep.cancer.gov/protocolDevelopment/electronic _applications/ctc.htm. Accessed 30 Oct 2019. 